\title{
Perspectivas teórico-metodológicas para el estudio de la vulnerabilidad social en los adultos mayores*
}

\section{Theoretical-methodological perspectives for the study of social vulnerability in the older adults}

\author{
Bernardino Jaciel Montoya Arce y \\ Alejandro Martínez Espinosa
}

\author{
Centro de Investigación y Estudios Avanzados de la Población \\ de la Universidad Autónoma del Estado de México
}

\section{Resumen}

En términos generales, se puede observar y a la vez decir, que como expresión de las contradicciones sociales se dan procesos de desigualdad y exclusión que colocan a los individuos ante situaciones de riesgo o riesgos según la situación que se analice y a esta condición se le ha dado en llamar vulnerabilidad social; este concepto en sí y por las múltiples dimensiones y complejidades requiere de definición epistemológica que le dé contenido conceptual; mismo que a la vez para su construcción y medición hace necesaria una metodología. La intensión de este artículo es hacer un ejercicio teórico-metodológico que en el mejor de los casos contribuya a aportar elementos para el estudio de la vulnerabilidad social en sus diversos aspectos y complejidades.

Palabras clave: Vulnerabilidad social, adultos mayores, índices.

\section{Abstract}

In general terms, it is possible to observe and comment that, as an expression of social contradictions, there are processes of inequality and exclusion that place individuals in situations of risk depending on the situation being analyzed and this condition has been called social vulnerability; this concept itself requires an epistemological definition that give it conceptual content due to multiple complexities and dimensions; in turn, a methodology is necessary to the construction and measurement of social vulnerability. The intention of this article is to make a theoreticalmethodological exercise that, in the best of cases, contributes to create elements for the study of social vulnerability in its various aspects and complexities.

Key words: Social vulnerability, older adults, index.

* Este artículo se elaboró como parte de las discusiones teórico-metodológicas que se llevan a cabo en el cuerpo académico "Procesos demográficos y política social", con clave de registro ante la Secretaría de Educación Pública: UAEM-CA-226 y nivel Consolidado. 


\section{INTRODUCCIÓN: LA UTILIDAD DEL CONCEPTO DE VULNERABILIDAD PARA} EL ANÁLISIS POBLACIONAL

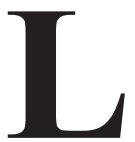

as posibilidades de que la vida se vea afectada por daños de diversa naturaleza ha sido una constante en la historia de la humanidad. Sin embargo, en tanto que esos daños pueden preverse, se han establecido múltiples formas de hacerles frente. Mediante la identificación de los riesgos, se hacía posible construir un andamiaje institucional para liberarse de la pobreza y de la dependencia (Beck, 1998). Ante el hecho de que los riesgos pueden amenazar a amplios sectores o grupos poblacionales, pero que no afectan a todos de la misma manera, el concepto de vulnerabilidad adquiere pertinencia, pues da cuenta del conjunto de condiciones que incrementa los riesgos de enfrentar un evento con consecuencias negativas (Welti, 2013).

El concepto vulnerabilidad tuvo como primer impulso analizar los impactos de los desastres naturales sobre las personas y sus comunidades (Cutter, 1996). Se trata de un concepto multidimensional, diferenciable entre contextos, que se relaciona con fragilidades, debilidades, susceptibilidades o falta de capacidades y resiliencia, que favorecen los efectos adversos diferenciados socialmente entre personas, grupos y comunidades (Tate, 2012).

Al tratarse de un concepto multidimensional, el análisis de la vulnerabilidad está sujeto a diversos métodos de medición (Chambers, 1989; Grundy, 2006). Por ejemplo, desde la Economía se consideran vulnerables a los hogares que pasan a un estado de pobreza resultado del proceso acumulativo de riesgo ${ }^{1}$ (Alwang, Siegel y Jorgensen, 2000). Desde la Demografía, la vulnerabilidad se define como el conjunto de características sociodemográficas vinculadas con las desventajas sociales y que están ligadas a la capacidad de movilizar activos ante los riesgos o amenazas (Rodríguez Vignoli, 2000). Como concepto previsor define la probabilidad de experimentar una pérdida en el futuro en relación con algún punto de referencia del bienestar. En este sentido, las personas en pobreza se consideran vulnerables si su acceso a bienes se encuentra limitado y sus capacidades para responder al riesgo son escasas. De manera similar, un hogar es vulnerable

\footnotetext{
${ }^{1}$ La vulnerabilidad inicia con la noción de riesgo. A la vez, el riesgo se caracteriza por una distribución de probabilidad conocida o desconocida de los acontecimientos que se caracterizan por su magnitud (incluyendo el tamaño y la extensión), su frecuencia y duración, lo cual afecta a los expuestos a vulnerabilidad. Los programas sociales pueden reducir el riesgo o la exposición al mismo (Alwang et al., 2001).
} 
si experimenta una pérdida de bienestar por eventos inciertos (Alwang et al., 2000).

Dentro de esa diversidad de aplicaciones, la vulnerabilidad se suele vincular con la capacidad de respuesta y resiliencia a los efectos negativos de un entorno cambiante (Chambers, 1989; Moser 1998). Así, la vulnerabilidad se asocia con la cantidad de activos que las personas, grupos o comunidades tienen para enfrentar dichos efectos negativos (Philip y Rayhan, 2004). La vulnerabilidad, entonces, puede ser analizada considerando dos extremos: una parte externa que se manifiesta mediante los riesgos, los choques y el estrés a los que se encuentra sujeto un individuo o un hogar; $\mathrm{y}$ una parte interna que se expresa a través de la ausencia de defensa, es decir, la carencia de mecanismos, recursos, habilidades o medios para hacer frente a la parte externa (Chambers, 1989). En general, la parte interna de la vulnerabilidad es la que más se analiza pues constituye la carencia de medios que las personas o grupos poseen para hacer frente a los riesgos y amenazas (Grundy, 2006).

En ese sentido, las diferencias entre perspectivas analíticas se asocian con los elementos del riesgo considerado, las opciones de respuesta para el manejo de los riesgos, así como los resultados expresados en pérdida de bienestar (Chambers, 1989; Shi y Stevens, 2005).

De acuerdo con la Comisión Económica para América Latina y el Caribe (CEPAL, 2002), la vulnerabilidad es considerada como un proceso en el cual puede encontrarse cualquier persona, grupo o comunidad que en un momento determinado pueda presentar una situación de desventaja, ${ }^{2}$ pero además de reconocer la importancia de los recursos para hacer frente a estas situaciones, agrega a los enfoques preexistentes el componente de la capacidad de adaptarse a los cambios.

El concepto de vulnerabilidad tiene un sentido preventivo, lo vulnerable no es lo vulnerado, sino una situación previa en la que aún se puede intervenir para impedir el daño (Ruiz, 2012). No obstante, la vulnerabilidad tiene un efecto espiral y vicioso, que hace que se potencien sus causas, que al padecer tal situación conduce a un estado cada vez más profundo de desmotivación, de discapacidad, de marginación, de pobreza, de exclusión social, etc., hasta que se consume la amenaza o riesgo produciéndose el daño que se buscaba evitar (Bueno y Diniz, 2008).

\footnotetext{
${ }^{2}$ El concepto de vulnerabilidad no debe confundirse con otros términos como pobreza, marginalidad, exclusión social, desafiliación, entre otros. Cada uno de éstos muestra características propias de un segmento de personas. Dichos conceptos no necesariamente son sinónimos, pero tampoco son mutuamente excluyentes o están desvinculados. Sin embargo, todos convergen en abordar aquellos grupos de población más desfavorecidos.
} 
La noción de vulnerabilidad viene delimitada en un tiempo y un espacio concreto, y acompañada por un "a qué" se es vulnerable. En la literatura especializada existe el consenso de que este fenómeno es multidimensional y afecta a individuos, familias, grupos o comunidades en distintos planos de bienestar, de diversas formas y con diferentes grados e intensidades (Chambers, 1989; Groba y Fustinoni, 2001; Bueno y Diniz, 2008; Ruiz, 2012; Tate, 2012; Otto, 2014).

Como se comentó, la vulnerabilidad se refiere al conjunto de condiciones de las personas, los hogares y las comunidades, que incrementan el riesgo de enfrentar un evento con consecuencias negativas. De modo que es fundamental tomar en cuenta que estos riesgos se presentan de manera desigual, de acuerdo con determinadas características sociodemográficas como la edad y el sexo, o según el contexto en el que se ubican las personas, así como por la incapacidad que tenga el individuo de cubrir sus necesidades básicas. En adelante profundizamos en los fuertes vínculos que existen entre la ubicación dentro de una determinada estructura social y los riesgos que pueden afectar la vida familiar y colectiva.

\section{VULNERABILIDAD SOCIAL Y POBREZA}

Para comenzar esta sección queremos enfatizar que muchos de los efectos negativos de los fenómenos naturales dependen de un gradiente de exposición, así como de las capacidades de recuperación y disponibilidad de recursos, que se reflejan finalmente en que los riesgos son socialmente diferenciados (Welti, 2013)

Atendiendo a esta particularidad, varias generaciones de especialistas han desarrollado el enfoque de la vulnerabilidad social para referirse a los aspectos sociales que determinan la vulnerabilidad de las personas y grupos desfavorecidos a distintos eventos negativos (Sánchez y Egea, 2011).

La construcción de ese concepto y recurso analítico, en América Latina y el Caribe (ALyC), ha sido resultado de una discusión bastante prolongada que tuvo su origen a finales de la década de los ochenta del siglo XX. Durante los años noventa, el concepto vulnerabilidad se emplea para analizar condiciones y situaciones desfavorables que se presentan como consecuencia de los efectos sociales de la década perdida (Oliver-Smith y Hoffman, 1999). Esto es, a partir de los ajustes estructurales y de la globalización, pero sobre todo por el cambio de modelo de desarrollo - el paso del modelo de Sustitución de Importaciones a un modelo Neoliberal-, se inicia a la desestructuración de diversos mecanismos institucionales para paliar los riesgos sociales, en consecuencia se erosiona de la seguridad 
social y el traslado de sus funciones a los ingresos laborales y a la familia (Ranci, 2009).

Cabe señalar que las condiciones que favorecen la distribución desigual de los riesgos y de sus consecuencias no es producto del periodo señalado; sin embargo, los desarrollos conceptuales previos resultaban limitados en cuanto a su capacidad explicativa.

Durante el periodo en el que la estrategia de desarrollo se basó en la Sustitución de Importaciones, uno de los marcos analíticos más recurrentes para analizar las condiciones sociales de los más desfavorecidos fue el de la marginalidad. Este concepto se forjó dentro de la teoría de la modernización, para tratar de comprender la base del "subdesarrollo" latinoamericano. La principal explicación sería la persistencia de vestigios de normas, valores y formas de ser de épocas pasadas, en un segmento tradicional que no permite que se desarrolle plenamente el segmento moderno de la sociedad. De modo que el problema era la personalidad marginal a la modernidad (Cortés, 2006).

Otra forma de entender ese fenómeno la proporcionó el enfoque histórico estructural, apoyado fuertemente en los supuestos marxistas y de la teoría de la dependencia. La población tiene escasas oportunidades de ser absorbida por el sistema productivo, de modo que se convierte en "ejército industrial de reserva", al margen del empleo estable y la protección social que proporciona la economía (Enríquez, 2007). Lo anterior establece una diferencia crucial con la perspectiva de marginalidad previa, al centrarse en las relaciones de producción propias del capitalismo dependiente. En lugar de enfocarse en el cambio de mentalidad, el énfasis se pone en las transformaciones requeridas para incorporar a la población al sistema productivo moderno (Cortés 2006).

Ambas perspectivas de marginalidad responden a las circunstancias propias del modelo de desarrollo vigente entonces, en el que grandes volúmenes de población se trasladaban a las ciudades dejando atrás los recursos materiales y sociales que permitían su subsistencia, sin contar con los requeridos en el ámbito urbano. Una vez que las tensiones sociales y macroeconómicas socavaron las bases del modelo de Sustitución de Importaciones, quedó claro la insuficiencia de ambas perspectivas para entender un conjunto de fenómenos emergentes.

Aunque la política económica se centró en el control de la inflación, esto no dio lugar a un crecimiento económico sostenido, además de permanecer por debajo del alcanzado entre la década de los cuarenta y los setenta del siglo XX. Por otro lado, el desempleo aumentó a la par de una 
menor velocidad en la reducción de la pobreza y una mayor desigualdad en términos de la distribución del ingreso. A fines de los noventa, la realidad social estaba configurada por una economía abierta pero poco competitiva, mientras que el Estado se había replegado de sus funciones productivas y sociales. Esto había dado como resultado que no sólo los estratos más empobrecidos vieran reducidas sus oportunidades para mejorar su situación, sino que los estratos medios se hallaran en indefensión e inseguridad, por lo que la vulnerabilidad se presenta como el rasgo característico de las sociedades latinoamericanas contemporáneas (Pizarro, 2001).

Tal ha sido el efecto de este enfoque que entre finales del siglo pasado y los primeros años del siglo XXI aún perdura, avanza y se perfila un marco teórico, debido a que éste logra captar y explicar los cambios experimentados que conducen a riesgos latentes que perjudican a amplios sectores de la población en ALyC (Sánchez y Egea, 2011, Kaztman, 1999; Moser, 1998).

Otro aspecto clave relacionado con la vulnerabilidad social, pero del que conviene identificar sus diferencias, es el de la pobreza. De hecho, considerarlos sinónimos implica que la vulnerabilidad no se analice adecuadamente e incluso se omita su incorporación en el diseño de políticas públicas (Chambers, 1989; Philip y Rayhan, 2004). La vulnerabilidad no se refiere a la pobreza como carencia de recursos materiales, sino que ésta contribuye a que las personas experimenten desventajas sociales con efectos negativos sobre la calidad de vida, la educación, el desarrollo de habilidades o la salud (Rogers, 1997; Zaidi, 2014; Nunes, 2016). En este sentido, la pobreza se refiere a la descripción de atributos que las personas o las familias comparten y que los perpetúan en situaciones de desventaja, mientras que la vulnerabilidad social se refiere a los procesos causales que sitúan a los individuos y/o sus familias en tales procesos (Pizarro, 2001). El papel de los recursos de los que se dispone, también puede contribuir a clarificar las diferencias sustantivas entre las nociones de "pobreza" y "vulnerabilidad social". Concretamente, analizar la pobreza suponía que los recursos disponibles fueran utilizados para como parte de la variable dependiente, mientras el estudio de la vulnerabilidad social propone el estudio de dichos recursos para la construcción de las variables independientes (Cepal, 2002).

Es importante destacar, que en el caso de los países de ALyC y como resultado de los procesos de exclusión social resultado de la implementación de las políticas neoliberales, el enfoque de la vulnerabilidad social comienza a replantearse ante el aumento del número de personas en situación de pobreza (Cadena Vargas, 2005; Álvarez Ayuso y Cadena Vargas, 2006). 
Como atinadamente lo citara Minujín (1999), ante la falta de políticas sociales de bienestar de carácter universal, el enfoque de la vulnerabilidad social comenzó un proceso de repunte.

Lo dicho hasta ahora permite reconocer que uno de los factores más relacionados con la vulnerabilidad social es la pobreza crónica y las desigualdades sociales, en tanto que la vulnerabilidad se manifiesta como parte de los procesos sociales que mantienen y reproducen las diferencias entre personas y grupos sociales (Sabates y Haddad, 2005). Asimismo da paso a señalar que los agregados de individuos (redes, familias) son vulnerables a condiciones sociales o políticas, a los procesos migratorios o los cambios en la estructura familiar o económica (Sánchez y Egea, 2011), así como por procesos de acumulación de riesgos relacionados con la salud (Nunes, 2016), entre otros. Dicha cuestión hace necesaria la reflexión en torno a la vulnerabilidad que se concentra en grupos sociales específicos.

\section{VULNERABILIDAD SOCIAL Y GRUPOS VULNERABLES}

La vulnerabilidad social enfatiza la capacidad de respuesta de personas, hogares o comunidades, según la disponibilidad de recursos, resiliencia, capital social y redes de apoyo institucionales y no institucionales (Birkmann, 2006). Es por esto que se puede identificar el efecto de la estructura social y económica a través de los grupos sociales, así como de las acciones y atributos de las redes y actores colectivos. Es en este sentido, que su estudio permite identificar fuentes de exclusión y reproducción de la pobreza, lo que se traduce en elementos clave para el diseño de la protección social (Sabates y Haddad, 2005).

Dentro del análisis de la vulnerabilidad social, los grupos vulnerables se definen en función de diversas características demográficas, sociales, económicas y políticas que incrementan la susceptibilidad al daño, resultado de la combinación de cambios en el curso de vida de las personas, hogares, comunidades o países y la disponibilidad de recursos para enfrentar tales condiciones (Grundy, 2006; Mechanic y Tanner, 2007). El término grupo vulnerable varía desde perspectivas como el feminismo o el marxismo. Desde la óptica feminista se define a las mujeres como vulnerables por las estructuras patriarcales subyacentes en las sociedades, mientras que desde el marxismo se argumenta que los bajos ingresos influyen en la salud de los trabajadores volviéndolos un grupo vulnerable (Larkin, 2009). Es decir, un grupo vulnerable es resultado de la articulación entre el riesgo de experimentar resultados negativos y la población en riesgo de padecer 
condiciones de salud, psicológicas o socioeconómicas adversas (Rogers, 1997; Sabates y Haddad, 2005; Schröder-Butterfill y Marianti, 2006).

Una de las características de las personas o grupos vulnerables es que éstos no cuentan con las capacidades para mantener la autonomía y poder tomar decisiones personales, por lo que son más propensas a experimentar daños reales o potenciales (Moore y Miller, 1999). Sin embargo, en contextos de subdesarrollo, el término grupo vulnerable adquiere sentido como una forma de focalizar la acción pública dada la reducción de la necesaria acción del Estado en materia de protección social (Aranibar, 2001), situación claramente notable para la mayoría de las naciones de ALyC (CEPAL, 2002).

En general, los grupos poblacionales que se consideran vulnerables son los niños, las mujeres, los adultos mayores, las personas con poca o nula escolaridad, los indígenas, los habitantes de las zonas rurales, los individuos con bajos ingresos o desempleados, los inmigrantes, los hogares con jefatura femenina, las personas con enfermedades crónicas o con el virus de inmunodeficiencia humana (VIH), o con problemas mentales severos (Rogers, 1997; Shi y Stevens, 2005).

\section{VULNERABILIDAd SOCIAL DE LAS PERSONAS MAYORES}

La situación anterior aunada a factores biológicos, psicológicos, sociales, culturales, económicos y políticos hace que los adultos mayores ${ }^{3}$ enfrenten condiciones de riesgo, que se traducen en diferentes tipos de vulnerabilidad: física-biológica, social-dependiente, ambiental-contextual (Sánchez, 2009b), demográfica (Cepal, 1999) y otras. En este sentido, toman importancia las dimensiones naturales y sociales de la vulnerabilidad, sobre todo la social sobre todo la social enmarcada por procesos macro y micro sociales que al atravesar los diferentes ámbitos de adscripción dan como resultado una construcción social compleja de desigualdades, falta de oportunidades, desprotección y otros factores cuya recuperación es necesaria para la comprensión de las condiciones de vida de estos individuos (Sánchez y Egea, 2011). De tal manera que se toma a la vulnerabilidad social como eje analítico para explicar el conjunto de riesgos sociales que enfrenta el grupo de personas de 60 años o más como: la alta fragilidad, la pérdida de calidad de vida, de salud, la reducción del ingreso, los problemas de vivienda, los cambios en la trayectoria laboral, la pérdida de cobertura social

\footnotetext{
${ }^{3}$ Es interesante que la definición de adulto mayor se basa en un atributo demográfico como la edad. En el caso de las sociedades desarrolladas el punto de corte es a los 65 o más años de edad, mientras que en las sociedades no desarrolladas se define una persona como adulto mayor a partir de los 60 años de edad (Organización Mundial de la Salud, 2015).
} 
y asistencial, los cambios en la estructura familiar, entre otros (Ruiz, 2012; González, 2009; Cepal, 2002).

Adicionalmente, las personas de edades avanzadas suelen presentar vulnerabilidad social como consecuencia de aspectos como el estado de salud, la capacidad cognitiva y el apoyo social. En este sentido, se sabe qué aspectos como la capacidad física y la memoria disminuidas o la falta de herramientas para la búsqueda de información en las personas mayores son elementos asociados con la vulnerabilidad social (Dolan, y Messen, 2012). Ciertas características individuales, reflejan los daños de la vida social, al tiempo que son obstáculos para la participación en la vida social.

Las desigualdades sociales y económicas hacen que este segmento poblacional enfrente riesgo de pérdida de calidad de vida, de salud, de recursos, de vínculos e incluso alteraciones culturales (Villagómez y Sánchez, 2014; Sánchez y Egea, 2011; Rodríguez Vignoli, 2000). Busso (2001) sostiene que el grupo poblacional de 60 años o más es el que se encuentra frente a condiciones sin precedentes que lo convierten en un grupo vulnerable, ya que este segmento presenta mayores condiciones de riesgo, debido a que cuenta con menores elementos para enfrentar la pobreza, la dependencia, el aislamiento social, el maltrato o la violencia y, por lo tanto, se trata de un grupo que requiere de un tratamiento especial y separado.

Al respecto Compán y Sánchez (2005) mencionan que la vulnerabilidad de la población anciana está asociada a la edad y el sexo. En el caso de las mujeres ancianas se ha encontrado que presentan una mayor dependencia asociada a los problemas de salud, presencia de discapacidad para la realización de las actividades de la vida cotidiana, así como una disminución del apoyo informal de familiares y que las pocas beneficiarias de recibir una pensión, ésta es con montos muy bajos (Sánchez, 2009a; Ham y González, 2008).

Asimismo, es importante reconocer que entre la población adulta mayor existen individuos vulnerables que necesitan apoyo tanto por parte de la sociedad en general como por parte del Estado. Ante esto, resulta crucial el análisis de los recursos que las personas en edad avanzada utilizan para enfrentar la situación de vulnerabilidad en la que se encuentran. Esto incluye las políticas públicas y los recursos familiares alrededor de este grupo.

De esta manera, por vulnerabilidad social se entiende como el conjunto de riesgos que enfrentan los adultos mayores asociados a factores socioeconómicos entre los que destacan: los ingresos, el contexto familiar, condiciones de salud, las condiciones de la vivienda, los servicios y el equipamiento de éstas, situaciones que influyen en su calidad de vida y los coloca 
en una situación de debilidad o desventaja respecto a otros. Por lo anterior, se acepta que la vulnerabilidad social en los adultos mayores no es atribuible a un sólo individuo, sino que está determinada de manera estructural.

También se admite que en la vulnerabilidad social existe una interrelación de condiciones que provienen de crisis económicas, sociales, cambios en el mercado laboral, reducción de los ingresos y del consumo, pérdida de cobertura y protección social, entre otros (Ruiz, 2012; González, 2009; Sánchez y Egea, 2011; Stern, 2004; Cepal, 2002; Banerrechea et al., 2002).

\section{Aproximaciones metodológicas para la medición}

\section{DE LA VULNERABILIDAD SOCIAL}

De acuerdo con lo que se ha señalado, la vulnerabilidad social es un fenómeno multidimensional, debido a la diversidad de esferas involucradas (económica, de salud, familiar y de vivienda), que en conjunto conducen a situaciones negativas sobre grupos sociales específicos como el de los adultos mayores. Dicha particularidad, supone una serie de retos en el momento de construir indicadores para dimensionar su magnitud y alcance.

Existe una serie de procedimientos para medir la vulnerabilidad social, por ejemplo, se puede hablar de una combinación entre las necesidades básicas insatisfechas y las líneas de pobreza (Pizarro, 2001; Álvarez Ayuso y Cadena Vargas, 2006). Sin embargo, la mayoría de mediciones de la vulnerabilidad social se basan en la construcción de indicadores sobre variables sociodemográficas o económicas (Willis y Fitton, 2016). Una aproximación comúnmente utilizada se define por el desarrollo de técnicas estadísticas de estandarización como el análisis de componentes principales (ACP) (Cutter, Boruff y Shirley, 2003; Álvarez Ayuso y Cadena Vargas, 2005; Schmidtlein et al., 2008; Armas y Gavris, 2013; Montoya et al., 2016). Entre las principales dimensiones empleadas como insumos para la definición de la vulnerabilidad social se encuentran la asociada con factores económicos, de la salud, de la vivienda, y las relacionadas con la estructura familiar y el apoyo y soporte social.

\section{Dimensión económica}

Bajo el entendido de que un número importante de personas experimentan el proceso de envejecimiento con todos los cambios que esta transición implica, no sólo biológicos sino sociales, es importante evidenciar que una de las características de esta etapa es el retiro del trabajo remunerado o el llamado retiro de la actividad económica (Solís, 1999). Sin embargo, esta 
ausencia en la actividad productiva resulta motivo de análisis y debate, ya que la vulnerabilidad en la que hoy se encuentran las personas en edades avanzadas lleva a postergar el retiro. La escasa cobertura de los sistemas de pensiones y el bajo monto de los estipendios, provoca que para muchas personas en edades de 60 años o más el retiro laboral no ocurra como era de esperarse (Pizarro, 2001).

En este sentido, resulta importante identificar las características de las actividades que realiza la población para obtener ingresos. Al respecto, una característica de la población envejecida es el que desarrolle empleos u ocupaciones en condiciones de precariedad laboral (Popolo, 2001; Pizarro, 2001). De acuerdo con el Instituto Nacional de Estadística y Geografía (INEGI), en el año 2010, 75 por ciento de los adultos mayores que trabajaba lo hacían en el sector informal y se estima que la cifra fue cercana a 90 por ciento en 2017. Según Wong y Aysa (2001), cuando se hace referencia al sector informal de la economía, se habla del medio en donde los trabajadores no gozan de un salario constante y suficiente; incluyendo también a los trabajadores por cuenta propia - excepto técnicos y profesionales - que forman parte de la economía informal al no ser trabajadores asalariados.

Frente a las crecientes restricciones para insertarse en el mercado de trabajo formal, donde una prioridad es incorporar a una creciente población en edad laboral, se construye una competencia tensa entre los diferentes segmentos poblacionales, con la finalidad última de ocupar espacios laborales restringidos, por lo que se hace en este sentido evidente la vulnerabilidad de la población envejecida ante la falta de oportunidades de empleo, pues se encuentra en una posición de desventaja evidente frente a otros grupos poblacionales (Wong y Aysa, 2001) quedando como único recurso la incorporación al autoempleo o al empleo informal (Ham, 1999).

Otros elementos asociados con el trabajo son: los ingresos y el acceso a las pensiones, aunque éstas en ciertas ocasiones sustituyen al ingreso laboral, no toda población cuenta con el privilegio. En términos generales, las pensiones tienen dos objetivos. Por un lado, mantener la calidad de vida en la vejez. Por el otro, retribuir la renta a lo largo de todo el ciclo de vida. En este contexto, la pensión se convierte en un elemento central de la vida de las personas mayores de 60 años, por lo que al no contar con un ingreso de este tipo coloca a los adultos mayores en riesgo de ser vulnerables al interior de la sociedad.

Entre los principales indicadores que suele incorporarse para determinar la vulnerabilidad social y que se relacionan con la dimensión económi- 
ca se encuentran: i) porcentaje de adultos mayores sin pensión o jubilación, ii) adultos mayores con ingresos menores a dos salarios mínimos, iii) porcentaje de personas mayores sin apoyo gubernamental (Álvarez Ayuso y Cadena Vargas, 2006; Montoya et al., 2016).

\section{Dimensión de salud}

De acuerdo con la Organización Mundial de la Salud (OMS), la salud se define como "un estado de completo de bienestar físico, mental y social y no solamente la ausencia de afecciones o enfermedades" (OMS, 1948). Se trata pues, de un concepto general que engloba o considera aspectos relacionados no solo con lo físico sino también, con elementos relacionados con la dimensión mental y social de las personas.

De esta manera, se asume que la vulnerabilidad en la salud es el conjunto de condiciones que, en función de características como la edad o el sexo, determinan un estado en el que los riesgos de enfrentar un evento con consecuencias negativas sobre el individuo se incrementan. Es importante mencionar que los riesgos pueden variar según el contexto en el que se ubica un individuo (Tate, 2012).

La vulnerabilidad en salud está determinada por la capacidad o la incapacidad del individuo de cubrir sus necesidades básicas. Para la determinación del grado de vulnerabilidad en la salud se consideran los factores que inciden en su deterioro físico y motriz como las discapacidades, que están relacionadas con la disminución en el grado de autonomía para satisfacer sus necesidades básicas, entre ellas el desplazamiento, toma de alimentos, ayuda para realizar su aseo en general e interacción con otras personas (Angelini Dos Santos e Iost Pavarini, 2011).

Otro elemento central en la salud de los adultos mayores es el acceso a la seguridad social, debido a que este mecanismo de protección garantiza la atención médica de la población mayor a 60 años, por lo que la seguridad social se convierte en un factor determinante para incidir positivamente sobre el bienestar de la población envejecida, lo que es una clara muestra de que el Estado debería atender y proteger a la sociedad y en especial a los grupos vulnerables (Pizarro, 2001).

Entre los principales indicadores citados en la literatura y que relacionan aspectos de la salud con la vulnerabilidad social de las personas mayores pueden citarse: a) porcentaje de adultos mayores con alguna limitación física o mental, b) porcentaje de adultos mayores que no tienen derecho a recibir servicios médicos y aquéllos que no se atienden en alguna institu- 
ción de salud (Angelini Dos Santos e Iost Pavarini, 2011; Montoya et al., 2016).

\section{Dimensión familiar}

La transición demográfica ha traído como consecuencia la disminución de la fecundidad, cambio que ha provocado un menor número de integrantes en las familias. Este hecho, que a simple vista podría parecer sencillo, no lo es pues tiene implicaciones en las funciones que los integrantes de ésta tendrán al interior del hogar en su futuro. Ante este hecho se considera importante incluir indicadores de familia en el estudio de la vulnerabilidad social del envejecimiento, ya que esta dimensión está relacionada con las redes de apoyo que la población adulta mayor puede o no tener al llegar a esta etapa de la vida (Montes de Oca y Hebrero, 2006).

Al respecto, el concepto de vulnerabilidad hace referencia a dos tipos de elementos; por una parte, los recursos disponibles, junto con las estrategias de su manejo (en grupos familiares o comunidades) y por otra, a la estructura de oportunidades que permiten su aprovechamiento. Concretamente, la vulnerabilidad es el resultado de la interacción entre dichos elementos frente a un conjunto de riesgos derivados del contexto (Bayón y Mier y Terán, 2010).

Dentro de los recursos que la población envejecida hace uso para enfrentar la vulnerabilidad, se encuentra la institución más importante de cualquier grupo social: la familia. En los grupos familiares en los que vive un anciano éste debe cumplir con ciertas funciones o labores para el grupo, en algunos casos es la cocinera, el cuidador de nietos o quizás uno de los proveedores del gasto común, ya sea por medio de su prolongación en el trabajo remunerado, de una pensión o de ayuda que recibe por otros medios (programas sociales o ayuda de otros parientes que no residen con él). En este sentido, es importante mostrar los elementos que dentro de un grupo familiar hacen vulnerables a los miembros envejecidos, y en su caso mostrar los recursos que la familia ofrece para enfrentar las desventajas que el grupo social representa (Angelini Dos Santos e Iost Pavarini, 2011).

En este marco, Ogg (2005) y Scharf et al., (2002) mencionan que la vulnerabilidad se deriva de las condiciones de exclusión social, que son el resultado de un ingreso insuficiente para satisfacer las necesidades básicas del hogar, malas condiciones de salud, carencia de interacción con otros individuos y un ambiente familiar inadecuado para vivir.

Con respecto a lo anterior, entre los principales indicadores que se incluyen como parte de la dimensión familiar de la vulnerabilidad social en 
las personas mayores se encuentran: i) porcentaje de adultos mayores que forman un hogar unipersonal, ii) porcentaje de personas que forman un hogar unipersonal (Angelini Dos Santos e Iost Pavarini, 2011; Montoya et al., 2016).

\section{Dimensión de vivienda}

Desde 1983 en México, por derecho constitucional todas las familias deben tener una vivienda digna y decorosa. No obstante, el Comité de Derechos Económicos, Sociales y Culturales de las Naciones Unidas (Naciones Unidas, 1991) menciona que no se trata sólo del derecho a la vivienda, sino a una vivienda adecuada, lo que tiene que ver con: i) seguridad jurídica de la tenencia; ii) disponibilidad de servicios materiales e infraestructura, iii) gastos adecuados según los ingresos percibidos; iv) habitabilidad; v) accesibilidad; vi) situarse en un lugar adecuado para permitir el acceso actividades, y vii) adecuación cultural de la vivienda. Si uno de estos puntos no se respeta, el derecho humano a la vivienda adecuada no se satisface.

A partir de lo anterior, esta dimensión comprende tanto la infraestructura de la vivienda como los bienes y servicios de ésta, con la idea de estimar el nivel de vulnerabilidad, riesgo e incapacidad que tiene la población adulta mayor cuando padece y sufre por los problemas derivados de las condiciones materiales de la vivienda, así como la ausencia de bienes y servicios dentro de ésta.

La posesión de activos en los hogares, lo que varios autores han denominado como estructuras de oportunidades, es determinante para definir la situación de vulnerabilidad social de los adultos mayores dentro de su hogar y su capacidad para salir de la pobreza. De acuerdo con Moser, Mcllwaine y Holland (1997) mientras mayor sea la cantidad de bienes y servicios mayor es el nivel de bienestar y la capacidad de reaccionar y mitigar los eventos negativos (Cervantes y Bueno, 2009; Ochoa, 2013). En este sentido, se han considerado las características de infraestructura de la vivienda de los adultos mayores, así como los bienes y servicios que cuentan sus hogares para su sobrevivencia diaria. Aunque habría que considerar la posibilidad de que ellos puedan operar esos recursos sobre todo tratándose de aparatos electrónicos.

Sobre lo anterior y considerando los indicadores comúnmente incluidos en esta dimensión son aquéllos que muestran las características materiales la vivienda o el número de bienes y servicios, y que generalmente se trata de: a) porcentaje de adultos mayores que habitan viviendas en piso de tierra, tienen un solo cuarto, sin luz eléctrica, sin sanitario, sin agua entubada, 
sin drenaje, sin refrigerador y sin lavadora (Álvarez Ayuso y Cadena Vargas, 2006; Montoya et al., 2016).

\section{Políticas públicas y Vulnerabilidad social}

\section{EN LOS ADULTOS MAYORES}

El término grupo vulnerable se desarrolla como una forma de focalizar la acción pública dada la reducción del papel del Estado en materia de protección y bienestar social sobre todo a partir de la implementación de las políticas económicas neoliberales. En particular, una de las principales objeciones en torno a la implementación de las políticas neoliberales ha sido la falta de la llamada "perspectiva universal" de las políticas estatales (Aranibar, 2001; Pizarro, 2001).

El concepto de política pública comenzó a acuñarse a finales del siglo XIX en los Estados Unidos. Sin embargo, su mayor desarrollo se presentó a partir de 1950, con la idea de que las Ciencias Sociales aportaran elementos para el diseño de éstas (Méndez, 1993). En torno a la noción de política pública se ha generado un debate en el cual no existe un consenso pleno sobre la definición de dicho concepto (Aguilar, 1991). En este sentido, González (2009) expone múltiples y diferenciadas definiciones de política pública, concluyendo aquélla que "implica el establecimiento de una o más estrategias orientadas a la resolución de problemas públicos, así como a la obtención de mayores niveles de bienestar social".

De acuerdo con Welti (2013) el concepto de política pública hace referencia al conjunto de acciones gubernamentales que buscan mejorar las condiciones de bienestar de los individuos, como la asignación de recursos para incrementar el acceso a los servicios de salud, de educación, de vivienda y de alimentación que garanticen al menos la sobrevivencia de quienes están más desprotegidos o en situación de vulnerabilidad, y que se ha considerado como un instrumento que sirve para garantizar el ejercicio de los derechos sociales.

Dentro del análisis de la política pública pueden destacarse cuatro tipos de políticas públicas: i) distributivas, ii) regulatorias, iii) redistributivas y iv) constitutivas. Las políticas distributivas son aquéllas que el Estado implementa con base a conductas o situaciones en la que se encuentran individuos sin establecer obligaciones; las políticas de subsidios constituyen un ejemplo de esta categoría. Mientras que las políticas regulatorias son implementadas por parte del Estado para intentar influir en el comportamiento y la conducta de los individuos, mediante el uso de sanciones por 
conductas transgresoras o por no respetar las reglas (Horbath y Gracia, 2010).

Por su parte las políticas redistributivas suponen la transferencia de recursos, a partir de la acción coercitiva del Estado; dentro de esta categoría se encuentran las políticas que otorgan beneficios o concesiones a ciertos individuos cuyos costos tendrán que ser financiados por otros. Las políticas constitutivas son aquellas acciones del Estado que apuntan a establecer las reglas a partir de las cuales se reparte el poder en un determinado marco societal, redefiniendo las condiciones del sistema político o administrativo. En esta categoría se incluyen las políticas orientadas a la creación de instituciones o agencias gubernamentales, así como aquéllas que apunten a generar nuevos marcos normativos (Horbath y Gracia, 2010).

Esta tipología se complementa identificando el grupo de población al cual van dirigidas las acciones gubernamentales. Dicho segmentado de población puede definirse por ciertas características estructurales: grupo etario, sexo, cultura, religión, entre otras o por compartir una serie de carencias donde destaca la pobreza, el desempleo, la desnutrición, entre otros (Horbath y Gracia, 2010).

En el caso de México, el debate sobre el concepto de política pública se ha centrado principalmente en clasificarlas como focalizadas y en pocos intentos como universales. Las primeras atienden grupos poblacionales específicos que comparten problemas singulares. Las segundas garantizan el ejercicio de derechos sociales a toda la población. Con el cambio de modelo económico que pasó de Sustitución de Importaciones a un modelo neoliberal, ha prevalecido el diseño de políticas públicas a partir de programas focalizados (Aguilar, 1991).

En este sentido, desde 1922 hasta al año 2000, el conjunto de acciones gubernamentales fue hacía políticas públicas distributivas, después de este año fueron focalizadas, argumentando la ineficacia de estas últimas (Kuntz, 2010). No obstante, todas las políticas públicas implementadas han atendido al segmento poblacional predominante. Es decir, desde principios del siglo pasado hasta los años setenta, las políticas públicas respondían a una pirámide poblacional joven, y las leyes, programas y organizaciones se orientaron a atender y modificar las condiciones de salud, de educación, de vivienda, de alimentación y de bienestar a este grupo de población, en especial de los niños y de las mujeres en edad reproductiva.

Posteriormente en el periodo de 1970 al año 2000, se presentaron nuevas situaciones económicas, sociales acompañadas de una estructura demográfica aun predominantemente joven, con otro tipo de necesidades 
que atender como educación, trabajo, salud, entre otras, lo anterior generó nuevas políticas públicas, sobre todo aquéllas que impulsaron los servicios educativos.

No obstante, a partir de siglo XXI las políticas públicas han tenido un cambio en su operacionalización como consecuencia de una estructura demográfica más envejecida. En especial, la focalización de políticas públicas para atender a la población adulta mayor, se debe por un lado al aumento de personas de 60 años o más, y por otro a la presión en el ámbito internacional por Organizaciones como las Naciones Unidas (1995) o la Cepal (2002) por atender a grupos en vulnerabilidad social.

Oficialmente la política social federal dirigida a las personas mayores de 60 años, comenzó en el año 2003 cuando la Secretaría de Desarrollo Social (SEDESOL) presentó el Programa de Atención a Adultos Mayores en Zonas Rurales, cuyo objetivo fue apoyar a este segmento de la población para lograr superar las condiciones de pobreza alimentaria en que se encontraban y acceder a mejores condiciones de vida. El programa funcionó así cuatro años. No obstante, en el año 2007 las reglas de operación se modificaron y a los adultos mayores de 60 a 69 años de edad los ubicaron en otros programas sociales debido a que representaban la mayor proporción de los adultos mayores, y generaban un monto de recursos que salía del presupuesto federal (Welti, 2013; SEDESOL, 2007).

Es así que en el año 2007 comienza a operar el Programa de Atención a los Adultos Mayores de 70 años o más residentes en zonas rurales. Este programa comenzó sólo para los residentes en localidades de hasta diez mil habitantes, y poco a poco se ha ido ampliando hasta atender a los adultos en que viven en localidades de hasta 30 mil habitantes. El Programa 70 y Más otorga apoyos monetarios bimestrales, jornadas informativas sobre temas de salud y servicios, así como apoyos del Instituto Nacional de las Personas Adultas Mayores (INAPAM), además de las que ofrecen actividades productivas, ocupacionales y culturales.

Para atender la carencia de seguridad social, ampliamente extendida en la población, en el año 2006, apareció el sistema de ahorro a trabajadores no asalariados y profesionistas independientes. Este Mecanismo de Ahorro para el Retiro Oportunidades (MAROP) se estableció como estrategia de ahorro en cuentas individuales que se constituía con las aportaciones de las personas y una aportación del Gobierno Federal. Sin embargo, fue eliminado dos años después. En el año 2008 se implementó el Programa de desarrollo humano Oportunidades, que apoyaba a toda la población en situación de pobreza, y por lo tanto estaban incluidos los adultos de 70 
años en adelante, a través del Programa de atención a adultos mayores de 70 años o más en zonas rurales. En el año 2014, el Programa de desarrollo humano oportunidades o Progresa se convirtió en Prospera.

A nivel subnacional, también surgieron programas que tuvieron como marco de referencia los programas del Gobierno Federal de atención a los adultos mayores de 70 años. En el año 2000, el entonces Distrito Federal dio a conocer la Ley de los Derechos de las Personas Adultas Mayores con residencia en dicho lugar, misma que sirvió como antecedente para la Ley nacional de los derechos de las personas adultas mayores en el año 2003. A partir de esta ley, se estableció el Programa de apoyo alimentario, atención médica y medicamentos gratuitos. En 2003, se dio a conocer la Ley que establece el derecho a la pensión alimentaria para los adultos mayores de setenta años mediante el cual surge una nueva concepción de política social para convertirla en un mecanismo a través del cual se ejercen los derechos sociales y se pasa de la asistencia a la participación social (Welti, 2013).

Sin embargo, es claro que el proceso de envejecimiento de la población abre nuevos escenarios en materia de política pública (Ham, 1999). Pues con el aumento de personas de 60 años o más se plantea nuevos retos para las instituciones que diseñan políticas, las cuales se traducen en programas sociales focalizados para atender algunos problemas que aqueja la población adulta mayor en México, por lo que la implementación de una política social será exitosa siempre y cuando logre mejorar las condiciones de vida de la población beneficiaria.

Finalmente, es necesario señalar las ventajas que representa considerar la vulnerabilidad social de los adultos mayores. Por un lado, se debe de tomar en cuenta que formar parte de este grupo etario no supone necesariamente que se encuentre en condiciones de desventajas sociales. Robles (2006), sugiere cuestionar la identificación que se hace entre vejez y dependencia considerando que en las edades mayores a los 75 años (cuarta edad) el deterioro suele ser más pronunciado que entre los mayores a 60 años. Para ello, la autora propone identificar fases de dependencia en la población adulta mayor, asumiendo que la necesidad de atención se concentra en las mayores edades.

Sin embargo, la limitación conceptual de asociar la dependencia a la vejez, no parece subsanarse identificando fases. La disponibilidad de ahorros que se pudieron haber realizado, los recursos institucionales que aseguren una pensión y servicios médicos, el acceso a vivienda, además de los apoyos familiares al alcance, marcan la diferencia en las condiciones de vida de los adultos mayores. De modo que participar de esos recursos sociales, 
permite llegar en mejores condiciones a la vejez. Es necesario tomar en cuenta que un mayor conjunto de desventajas experimentadas en las edades previas haría más probable que las personas experimenten daños y vieran comprometida su independencia aun antes de la llamada cuarta edad. Es en este sentido que la identificación de la vulnerabilidad social, más que la edad o la etapa de la vejez en la que se encuentren las personas provee de insumos conceptuales y operativos para entender la heterogeneidad que marca el proceso de envejecimiento, a la vez que permite enfocar los esfuerzos institucionales para su adecuada atención.

\section{Conclusión}

En su definición amplia, el concepto de vulnerabilidad hace referencia a las debilidades o falta de capacidades y de resiliencia que favorecen los efectos negativos de las dinámicas del contexto por lo cual sus consecuencias son socialmente diferenciadas (Welti, 2013). En este sentido, el concepto vulnerabilidad social se refiere a los aspectos sociales que determinan la vulnerabilidad de las personas y grupos desfavorecidos a eventos negativos (Sánchez-González y Egea-Jiménez, 2011). De hecho, la construcción de este recurso analítico se ha visto orientado por diversos enfoques entre los cuales destacan el enfoque de activos-estructura de oportunidades o el enfoque histórico estructural que enfatizan la carencia de oportunidades en el contexto de los cambios macroeconómicos como los experimentados en las sociedades de ALyC.

Si bien suele confundirse e intercambiarse con el concepto de pobreza, es importante que en el análisis de la vulnerabilidad social se identifiquen plenamente sus diferencias. Este podría ser el caso de los estudios enfocados al análisis de las características de los grupos vulnerables entre los que destaca el de los adultos mayores.

En el contexto de envejecimiento de las estructuras etarias de los países de ALyC, el análisis de la vulnerabilidad social se perfila como un elemento deseable en la búsqueda de una mayor comprensión de los mecanismos y las estructuras que propician los efectos negativos del entorno sobre la población adulta mayor.

Dentro del área de estudio de la Demografía y los Estudios de Población, es importante considerar que la vulnerabilidad social de los adultos mayores se presenta como resultado de una construcción social compleja que se reproduce a través de múltiples mecanismos de desigualdad, desprotección o carencia de oportunidades. Al respecto, la aproximación que proporciona la vulnerabilidad social en tanto eje analítico, así como de las 
distintas aproximaciones metodológicas del análisis, se presentan como elementos que permitirían una mejor o mayor comprensión de determinados procesos que se relacionan con el envejecimiento, a saber: la disminución de la calidad de vida, de salud, el incremento de exposiciones a factores de riesgo en la salud, la carencia o problemas en la propiedad de la vivienda, la reducción del ingreso o la falta de pensiones, la pérdida de cobertura social y asistencial o los cambios en la estructura familiar, por sólo mencionar algunos.

Sin embargo, es claro que las dinámicas propias de las sociedades, aunadas a los procesos económicos imperantes en ALyC suponen otros retos sobre los cuales la perspectiva de la vulnerabilidad social podría ser considerada como opción analítica. En este sentido, no puede dejar de mencionarse la trascendencia de cuestionar no solo la perspectiva analítica adoptada, sino también las críticas hacia los indicadores construidos mediante los cuales se ha buscado captar los efectos de la vulnerabilidad social en los adultos mayores. Particularmente, en el caso de las mediciones que, basadas en las necesidades básicas insatisfechas o las líneas de pobreza intentan capturar la complejidad de la vulnerabilidad social, si bien la mayoría solo recupera variables sociodemográficas o económicas. De ahí que se destaque la importancia de la inclusión de factores económicos, de la salud, de la vivienda, y las relacionadas con la estructura familiar o el apoyo y soporte social en la definición y análisis de vulnerabilidad social de los adultos mayores.

\section{REFERENCIAS BIBLIOGRÁFICAS}

Aguilar, L., 1991, El estudio de las políticas públicas. Colección Antologías de Política Pública. México: Porrúa.

Álvarez Ayusso, I., y Cadena Vargas, E., 2006, "Índice de vulnerabilidad social en los países de la OCDE”, en Quivera, 8(2), pp. 248-274. Disponible en http:// www.redalyc.org/

Alwang, J., Siegel, P. y Jorgensen, S., 2000, Vulnerability: a view from different disciplines, Social protection discussion paper series. Washington: Banco Mundial.

Angelini dos Santos, A., y Iost Pavarini, S. C., 2011, "Functionality of elderly people with cognitive impairments in different contexts of social vulnerability", en Acta Paulista de Enfermagem, 24(49), pp. 520-526. Disponible en http://dx.doi. org/10.1590/S0103 21002011000400012

Aranibar, P, 2001, Acercamiento conceptual a la situación del adulto mayor en América Latina. Serie Población y Desarrollo, 21. CEPAL: Santiago de Chile. 
Armas, I., y Gavris, A., 2013, "Social vulnerability assessment using spatial multi-criteria analysis (SEVI model) and the Social Vunerability Index (SoVI model) - a case study for Bucharest, Romania", en Natural Hazards and Earth System Sciences, 13, pp. 1481-1499. Disponible en https://pdfs.semanticscholar. org/

Barrenechea, J., Gentile, S., González, S. Natenzon, C., y Ríos, D., 2002, Revisión del concepto de vulnerabilidad social, Pirna, Buenos Aires, Argentina.

Bayón, C., y Mier, M., 2010, Familia y vulnerabilidad en México: realidades y percepciones. México: UNAM, Instituto de Investigaciones Sociales.

Beck, U., 1998, La sociedad del riesgo: hacia una nueva modernidad. Madrid, Paidós.

Birkmann, J., 2006, "Indicators and criteria for measuring vulnerability: theoretical bases and requirements", en Birkmann, J, Measuring vulnerability to natural hazards: towards disaster resilient societies (pp. 55-77). Tokio: United Nations University Press.

Bueno, E. y Diniz, J.E., 2008, Pobreza y Vulnerabilidad Social: Enfoques y Perspectivas. Serie Investigaciones 3. Río de Janeiro:Asociación Latinoamericana de Población.

Busso, G., 2001, Vulnerabilidad social: nociones e implicaciones de políticas para Latinoamérica a inicios del siglo XXI. CEPAL, Informe de la Reunión de Expertos: Seminario Internacional sobre las diferentes Expresiones de la Vulnerabilidad Social en América Latina y el Caribe, División de Población de la CEPAL/ CELADE, Santiago de Chile, Chile.

Cadena Vargas, E., 2005, “El neoliberalismo en México: saldos económicos y sociales", en Quivera, 7(1), pp. 198-236. Disponible en http://www.redalyc.org/

CEPAL, 1999, Envejecimiento demográfico de México: retos y perspectivas. MéXico.

CEPAL, 2002, Vulnerabilidad sociodemográfica: Viejos y nuevos riesgos para comunidades, hogares y personas, División de Población de la CEPAL/CELADE, Santiago de Chile, Chile.

Cervantes, D., y Bueno, E., 2009, Vulnerabilidad social municipal en el Estado de Zacatecas, México", en Cuadernos Geográficos (45), pp. 173-207. Disponible en http://www.redalyc.org/

Chambers, R., 1989, Vulnerability: How do poor cope? IDS Bulletin, Sussex.

Compán D., y Sánchez, D., 2005, “Los ancianos al desván: El proceso de degradación biológica y social de la población mayor del municipio de Granada", en Cuadernos Geográficos, 36, pp. 25-274. Disponible en http://www.redalyc.org/

Cortés, F., 2006, “Consideraciones sobre la marginación, la marginalidad, marginalidad económica y exclusión social”, en Papeles de población, 12(47), 71-84.

Cutter, S. L., 1996, "Vulnerability to environmental hazards", en Progress in Human Geography, 20(4), 529-539. 
Cutter, S., Boruff, B. J., y Shirley, W., 2003, "Social vulnerability to environmental hazards", en Social Science Quarterly, 84(2), pp. 242-261. Disponible en https://onlinelibrary.wiley.com/doi/abs/10.1111/1540-6237.8402002

Dolan, G., y Messen, D., 2012, “Social vulnerability: An emergency managers' planning tool", en Journal of Emergency Management, 10(3), 161- 169. Disponible en https://www.wmpllc.org/ojs-2.4.2/index.php/jem/article/view/1246

Enríquez, P. G., 2007, "De la marginalidad a la exclusión social: un mapa para recorrer sus conceptos y núcleos problemáticos", en Fundamentos en humanidades, 8(15), pp. 57-88.

González, L., 2009, Vulnerabilidad social y dinámica demográfica en Argentina, 2001-07”, en Cuadernos Geográficos, (45), pp. 209-229. Disponible en https:// www.researchgate.net/publication/

Groba, G. y Fustinoni, A. M., 2001, Población vulnerable en la Ciudad de Buenos Aires, Hogares de día para la tercera edad. Centro de Documentación en Políticas Sociales.

Grundy, E., 2006, "Ageing and vulnerable elderly people: European perspectives", en Ageing and Society, 26(1), pp. 105-134.

Ham, R., 1999, "El envejecimiento en México: de los conceptos a las necesidades", en Papeles de Población 5(19), pp. 7-21. Disponible en http://www.redalyc. org/

Ham, R., y González, C., 2008, "Discriminación en las edades avanzadas en México", en Papeles de Población, 14(55), pp. 35-58. Disponible en http://www. scielo.org.mx/

Horbath, J., y Gracia, A., 2010, "Los desafíos del envejecimiento poblacional para las políticas públicas en México", en Revista de Relaciones Internacionales, Estrategia y Seguridad, 5(2), pp. 139-159. Disponible en http://www.redalyc.org/

Katzman, R., 1999, Activos y estructuras de oportunidades. Estudios sobre las raíces de la vulnerabilidad en Uruguay. Documento preparado por la Oficina de CEPAL en Montevideo, con el apoyo financiero del PNUD, en el marco del Proyecto URU/97/017 “Apoyo a la implementación del Programa de Acción de la Cumbre Mundial sobre Desarrollo Social". Montevideo: CEPAL.

Kuntz, S., 2010, Historia económica general de México. De la Colonia a nuestros días. México, El Colegio de México/Secretaría de Economía.

Larkin, M., 2009, Vulnerable Groups in Health and Social Care. London: SAGE publications.

Mechanic, D., y Tanner, J., 2007, "Vulnerable people, groups, and populations: Societal view", en Health Affairs, 26(5), pp. 1220-1230. Disponible en http://content.healthaffairs.org/content/26/5/1220.full.pdf + html

Méndez, J., 1993, La politica pública como variable dependiente: Hacia un análisis más integral de las políticas públicas. Lecturas básicas de administración y políticas públicas. El Colegio de México, México. 
Minujín, A., 1999, “¿La gran exclusión? Vulnerabilidad y exclusión en América Latina”, en Filmun, D. (ed.). Los noventa. Política, sociedad y cultura en América Latina y Argentina de fin de siglo. Buenos Aires: Eudeba.

Montes de Oca, V., y Hebrero, M., 2006, "Eventos cruciales y ciclos familiares avanzados: el efecto del envejecimiento en los hogares de México", en Papeles de Población, 12(50), 1-19. Disponible en http://www.scielo.org.mx/

Montoya, J., Román, Gaxiola, S., y Montes de Oca, H., 2016, “Envejecimiento y vulnerabilidad social en el Estado de México, 2010", en Papeles de Población, 22(90), pp. 43-77. Disponible en http://www.redalyc.org/articulo. oa? id=11249884003

Moore, L. W. y Miller, M., 1999, Initiating research with doubly vulnerable populations, en Journal of Advanced Nursing, 30(5), pp. 1034-1040.

Moser, C., 1998, “The asset vulnerability framework: reassessing urban poverty reduction strategies", en World Development, 26(1), pp. 1-9. Disponible en https:// www.sciencedirect.com/science/article/abs/pii/S0305750X97100158

Moser, C., McIlwaine, C., y Holland, J., 1997, Household responses to poverty and vulnerability - volume 4: confronting crisis in Chawama, Lusaka, Zambia. Urban management programme policy paper no. 24. Washington: Banco Mundial. Disponible en

Naciones Unidas, 1991, El derecho a una vivienda adecuada. Folleto informativo núm. 21/Rev. 1. ONU Habitat.

Naciones Unidas, 1995, The Copenhagen Declaration and Programme of Action: World Summit for Social Development. Nueva York: Naciones Unidas. Programa de las Naciones Unidas para el Desarrollo.

Nunes, A., 2016, Assets for health: linking vulnerability, resilience and adaptation to climate change. Tyndall Centre for Climate Change Research. Working Paper 163. Disponible en http://www.tyndall.ac.uk/sites/default/files/publications/ twp163.pdf

Ochoa, S. M., 2013, Riesgo y Vulnerabilidad laboral durante la crisis financiera y económica de 2008-2009 en México. Tesis para obtener el grado de Doctor en Ciencia Social con especialidad en Sociología, El Colegio de México.

Ogg, J., 2005, "Social exclusion and insecurity among older Europeans: the influence of welfare regimes", en Ageing \& Society, 25, pp. 69-90. Disponible en https://www.cambridge.org/

Oliver-Smith, A. y S. Hoffman, 1999, The angry earth: disaster in anthropological perspective. New York: Routledge.

Organización Mundial de la Salud, 1948, Preámbulo de la Constitución de la Organización Mundial de la Salud, que fue adoptada por la Conferencia Sanitaria Internacional. Génova: Organización Mundial de la Salud.

Organización Mundial de la Salud, 2015, Informe Mundial sobre el Envejecimiento y la Salud. Génova: Organización Mundial de la Salud. 
Otto, E., 2014, "Riesgo social: medición de la vulnerabilidad en grupos focalizados", en Cuadernos del Cimbage, (16), pp. 27-51. Disponible en http://www. redalyc.org/

Philip, D., y Rayhan, I., 2004, Vulnerability and poverty: what are the causes and how are they related?, Center for Development Research. Disponible en https:// www.zef.de/fileadmin/.../2004_3a_Philip_Rayan.pdf

Pizarro, R., 2001, La vulnerabilidad social y sus desafios: una mirada desde América Latina. Santiago de Chile: Naciones Unidas.

Popolo del, F., 2011, Características sociodemográficas y socioeconómicas de las personas de edad en América Latina. CEPAL. Disponible en https://www.cepal. org/

Ranci, C., 2009, Social vulnerability in Europe: The new configuration of social risks. Springer.

Robles Silva, L., 2006, "La vejez: nuevos actores, relaciones sociales y demandas políticas", en Relaciones. Estudios de historia y sociedad, 27(105), pp. 140-175. Disponible en http://www.redalyc.org/articulo.oa?id=13710505

Rodríguez Vignoli, J., 2000, Vulnerabilidad y grupos vulnerables: un marco de referencia conceptual mirando a los jóvenes, Comisión Económica para América Latina y El Caribe, Naciones Unidas, Santiago de Chile, Chile.

Rogers, A., 1997, "Vulnerability, health, and healthcare", en Journal of Advanced Nursing, 26, pp. 65-72. Disponible en https://onlinelibrary.wiley.com/doi/ abs/10.1046/j.1365-2648.1997.1997026065.x

Ruiz, N., 2012, "La definición y medición de la vulnerabilidad social. Un enfoque normativo", en Investigaciones Geográficas, Boletín del Instituto de Geografia, (77), pp. 63-74. Disponible en http://www.redalyc.org/articulo. oa? id $=56923353006$

Sabates, R., y Haddad, L., 2005, Reconciling different concepts of risk and vulnerability: areview of donor documents. Institute of Development Studies, Sussex.

Sánchez, D. y Egea, C., 2011, "Un enfoque de vulnerabilidad social para investigar las desventajas socioambientales. Su aplicación en el estudio de los adultos mayores", en Papeles de Población, 17(69), pp. 152-184. Disponible en http:// www.scielo.org.mx/

Sánchez, D., 2009a, "Contexto ambiental y experiencia espacial de envejecer en el lugar: el caso de Granada", en Papeles de Población, 15(60), pp. 175-213. Disponible en http://www.redalyc.org/articulo.oa?id=11211340008

Sánchez, D., 2009b, "Geografía del envejecimiento vulnerable y su contexto ambiental en la ciudad de Granada: Discapacidad, dependencia y exclusión social", en Cuadernos Geográficos, 2(45). Disponible en http://revistaseug.ugr.es/

Scharf, T., Smith, C. y Kingston, P., 2002, Growing older in socially deprived areas: social exclusion in later life. Londres: Help the Aged.ORG. 
Schmidtlein, M. C., Deutsch, R. C., Piegorsch, W. W. y Cutter, S. L., 2008, “A sensitivity analysis of the social vulnerability index", Risk Analysis: An International Journal, 28(4), 1099-1114.

Schröder, E., y Marianti, R., 2006, A Framework for understanding old-age vulnerabilities. Ageing and Society, 26(1), pp. 9-35. Disponible en https://www.ncbi. nlm.nih.gov/

SEDESOL, 2007, Reglas de operación de Sedesol. Secretaría de Desarrollo Social. Programa de Atención a Adultos Mayores en Zonas Rurales.

Shi, L. y Stevens, G. D., 2005, "Vulnerability and unmet health care needs: the influence of multiple risk factors", en Journal of general internal medicine, 20(2), pp. 148-154.

Solís, P., 1999, "La población en edades avanzadas”, en Gómez de León, J. y Rabell Romero, C. (ed.). La población de México. Tendencias y perspectivas sociodemográficas hacia el siglo XXI. México: FCE.

Stern, C., 2004, "Vulnerabilidad social y embarazo adolescente en México", en Papeles de Población, 10(39), pp. 129-158. Disponible en http://www.scielo.org. $\mathrm{mx} /$

Tate, E., 2012, "Social vulnerability indices: a comparative assessment using uncertainty and sensitivity analysis", en Natural Hazards, 63(2), pp. 325-347. Disponible en https://link.springer.com/article/10.1007/s11069-012-0152-2

Villagómez, G., y Sánchez, N., 2014, Mujeres mayas: envejecimiento, pobreza y vulnerabilidad", en Península, 9(2), pp. 75-98. Disponible en http://www.scielo. org.mx/

Welti, C., 2013, "Política social y envejecimiento", en Papeles de Población, 19(77), pp. 25-59. Disponible en http://www.redalyc.org/

Willis, I., y Fitton, J, 2016, "A review of multivariate social vulnerability methodologies: A case study of the River Parrett catchment", en Natural Hazards and Earth System Sciences, 16(6), UK. 1387-1399. https://doi.org/10.5194/ nhess-16-1387-2016

Wong, R., y Aysa, M., 2001, "Envejecimiento y salud en México: un enfoque integrado", en Estudios Demográficos y Urbanos, 16(3), pp. 519-544. Disponible en https://estudiosdemograficosyurbanos.colmex.mx/index.php/edu/article/ view/1107

Zaidi, A., 2014, Life cycle transitions and vulnerability in old age: a review. UNDP Human Development Report Office. Disponible en http://hdr.undp.org/ sites/default/files/hdr_2014_zaidi_final.pdf 


\section{RESUMEN CURRICULAR DE LOS AUTORES}

Bernardino Jaciel Montoya Arce

Doctor en Sociología por la Universidad Nacional Autónoma de México (UNAM). Pertenece al Sistema Nacional de Investigadores Nivel I y cuenta con perfil PROMEP. Actualmente es el coordinador del Centro de Investigación y estudios Avanzados de la Población de la Universidad Autónoma del Estado de México (CIEAP/UAEM). Entre sus publicaciones recientes se encuentran Demografia indígena en el Estado de México (coautor), 2013; Análisis demográfico del envejecimiento en el Estado de México (compilador), UAEM, 2011 y "La educación indígena en el Estado de México", en Papeles de Población núm. 75, 2013.

Dirección electrónica: bjmontoyaa@uaemex.mx

\section{Alejandro Martínez Espinosa}

Sociólogo por la UNAM, maestro en Estudios de Población por El Colegio de la Frontera Norte y Doctor en Estudios de Población por El Colegio de México. Investigador posdoctoral en El Colegio de la Frontera Norte entre 2017 y 2018. En los artículos de su autoría se abordan los cambios contextuales, así como las características de los hogares, en su relación a las prácticas alimentarias de la población. Dirección electrónica: amartineze@uaemex.mx

Artículo recibido el 15 de febrero de 2018 y aprobado el 26 de septiembre de 2018 . 